Second- and third-harmonic parametric scattering in disordered quadratic media

This article has been downloaded from IOPscience. Please scroll down to see the full text article.

2010 J. Phys. B: At. Mol. Opt. Phys. 43215404

(http://iopscience.iop.org/0953-4075/43/21/215404)

View the table of contents for this issue, or go to the journal homepage for more

Download details:

IP Address: 130.56.65.24

The article was downloaded on 13/12/2010 at 23:23

Please note that terms and conditions apply. 


\title{
Second- and third-harmonic parametric scattering in disordered quadratic media
}

\author{
Wenjie Wang ${ }^{1,2}$, Ksawery Kalinowski ${ }^{1}$, Vito Roppo ${ }^{1,3}$, Yan Sheng ${ }^{1}$, \\ Kaloian Koynov ${ }^{4}$, Yongfa Kong ${ }^{2}$, Crina Cojocaru ${ }^{3}$, Jose Trull ${ }^{3}$, \\ Ramon Vilaseca $^{3}$ and Wieslaw Krolikowski ${ }^{1}$ \\ 1 Nonlinear Physics Center and Laser Physics Center, Research School of Physics and Engineering, \\ Australian National University, Canberra ACT 0200, Australia \\ 2 School of Physics, Nankai University, Tianjin, 300071, People's Republic of China \\ 3 Departament de Fisica i Enginyeria Nuclear, Escola Tecnica Superior d'Enginyeries Industrial y \\ Aeronautica de Terrassa, Universitat Politecnica de Catalunya, Colom 11, 08222 Terrassa, Barcelona, \\ Spain \\ 4 Max-Planck Institute for Polymer Research, Ackermannweg 10,55128, Mainz, Germany \\ E-mail: joywwj@gmail.com
}

Received 1 July 2010, in final form 27 September 2010

Published 22 October 2010

Online at stacks.iop.org/JPhysB/43/215404

\begin{abstract}
We study frequency conversion via interaction of two fundamental beams in quadratic nonlinear media with random distribution of the ferroelectric domains. We demonstrate broadband second- and third-harmonic generation, analyse both spatial and polarization properties of the emitted harmonics and show that they closely follow the theoretical predictions.
\end{abstract}

(Some figures in this article are in colour only in the electronic version)

\section{Introduction}

There is a natural common perception that coherent optical phenomena are always intimately associated with perfectly ordered media as is, for instance, the case for normal light propagation in crystals where particular ordering of atoms or ions and spatial symmetry associated with this ordering defines particular optical properties including the nonlinear optical response. In fact, the lack of this ordering immediately precludes certain optical properties from being observed. However it turns out that this is not always true and actually introducing disorder into an otherwise perfect optical system (structure) is in fact beneficial. As BaudrierRaybaut et al [1] demonstrated recently the nonlinear optical process of non-phase-matched frequency conversion is much more efficient when realized in a polycrystalline medium consisting of micron-size randomly oriented crystallites than the same process conducted in a regular crystal of the same material. This beneficial role of disorder on the parametric process has been confirmed in a number of other works in recent years. Particularly interesting in this context is the use of ferroelectric crystals with disordered domain structure. Already Horowitz et al [2] and Kawai et al [3] have shown that as-grown strontium barium niobate (SBN) crystals could be used to realize broadband second and sum-frequency generation. This was possible due to the presence of elongated antiparallel ferroelectric domains which leads to spatial random modulation of the sign of the quadratic nonlinearity. Such nonlinearity modulation provides a continuous domain of reciprocal wave vectors $\vec{G}$ which enable us to fulfil the phase-matching condition [4] which for second-harmonic generation (SHG) reads $2 \vec{k}_{(\omega)}+\vec{G}=\vec{k}_{(2 \omega)}$, where $\vec{k}_{(\omega)}$ and $\vec{k}_{(2 \omega)}$ are the wave vectors of the fundamental (FW) and the second-harmonic (SH) beams, respectively. It appears that the multi-domain SBN crystal is particularly well suited for the broadband operation because its spatial random distribution of nonlinearity enables phase matching for the second-order nonlinear processes at practically any incident wavelength [5]. A number of useful applications have been demonstrated with this crystal including e.g. multi-frequency conversion [6-8], conical and planar emission [9-11] as well as the frequency mapping from infrared to visible [12]. It was also shown that the SBN crystal with disordered domains can be used to construct simple optical auto-correlator [11, 13]. It has been also recently demonstrated that such nonlinear 


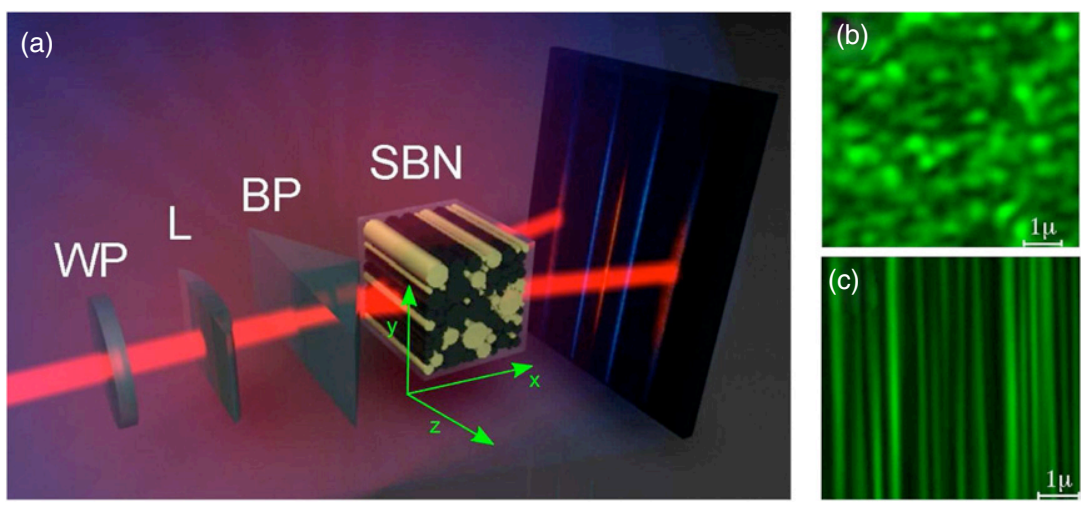

Figure 1. (a) Schematic of the experimental setup. The multi-domain structure of the SBN sample is schematically shown. WP-half-wave plate, L-cylindrical lens, BP-bi-prism. (b and c) Multi-domain structure of the SBN sample as visualized by the second-harmonic nonlinear microscopy; viewing area perpendicular (b) and parallel (c) to the optical $(Z)$ axis.

medium with random domains can support cascaded thirdharmonic generation $[5,14,15]$.

As far as the application for pulse monitoring and characterization is concerned, the nonlinear interaction in the disordered crystal involves two beams (pulses) intersecting at a small angle inside the disordered medium. We have recently investigated this interaction scenario in the context of SHG [10] using fundamental beams from the very near-infrared part of the spectrum (1064 nm and less). In this work we extend our studies by considering simultaneous second- and third-harmonic generation via cascading. This is particularly relevant for interactions involving fundamental waves at the telecommunication wavelengths as the cascading leads to the emission of the third harmonic in the visible part of the spectrum, far from the absorption edge of the commonly used quadratic crystals. We investigate the polarizational properties of the emitted harmonics and model them using the concept of effective nonlinearity and taking into account the role of spatial randomness of the antiparallel ferroelectric domains. We support our experimental results with direct simulations of the Maxwell equation in a random quadratic medium.

\section{Experimental setup}

The experimental setup is shown in figure 1(a). As a light source we used an optical parametric amplifier (TOPAS) generating $150 \mathrm{fs}$ pulses ( $\lambda=1450 \mathrm{~nm}, 250 \mathrm{~Hz}$ rep rate) with total energy of $0.788 \mathrm{~mJ}$ and beam diameter $5 \mathrm{~mm}$ (full width at half maximum). The beam was propagating through the half-wave plate (WP) in order to control its polarization, and then after focusing with a $100 \mathrm{~mm}$ cylindrical lens (L) was incident at the bi-prism (BP). Bi-prism converted the incoming beam into two symmetric beams which then intersected at the angle of $2 \times \alpha=2 \times 2.344^{\circ}$ inside the SBN crystal with a random distribution of the antiparallel ferroelectric domains. This random domain pattern is illustrated in figures 1(b) and (c). These graphs were obtained by using the SH nonlinear microscopy [16] and depict the domain structure as seen along (figure 1(b)) and perpendicular (figure 1(c)) to the optical axis which coincides with the longer dimension of the domains. In the experiment, the orientation of the crystal was such that both beams propagated in the $X-Z$ plane, close to its crystallographic $X$-axis and nearly perpendicularly to the longer dimension of the domains (coinciding with the optical axis), as illustrated in figure 1(a). Interaction of these two beams inside the crystal resulted in the emission of second- and third-harmonic waves whose far field in the forward direction was recorded using a CCD camera located behind the SBN crystal.

In the experiment the input polarization of the fundamental beam was varied from ordinary to extraordinary by adjusting the WP. Band-pass filters (not shown) placed in front of the CCD camera were used to separate the fundamental beam and its harmonics so that their corresponding spatial intensity distribution could be separately recorded. In addition, a polarizer (not shown) located behind the SBN sample enabled measurements of the ordinary and the extraordinary components of the generated harmonics.

\section{Experimental results}

When the two intersecting linearly polarized fundamental beams propagate in the sample of the SBN crystal, the nonlinear interaction in the crystal leads to the emission of both second and third harmonics. Unlike our previous works with the fundamental wavelength of $1.064 \mu \mathrm{m}$ or $0.8 \mu \mathrm{m}$ when the third harmonic could not be observed since it was above the absorbtion edge of the medium, here it was in visible part of the spectrum and its intensity was high enough to be measured and characterized. Because of the randomness the overall generated signals were rather weak. The total emitted power of the second and third harmonics reached $35 \mu \mathrm{W}$ and $16 \mathrm{nW}$, respectively. A typical harmonic intensity pattern observed on the screen is depicted in figure 2. It consists of seven lines oriented perpendicularly to the optical $(z)$ axis of the crystal. The three lines denoted as $\mathrm{SH} 1, \mathrm{SH} 2$ represent the second harmonic while the four lines marked as TH1 and TH2 represent the third-harmonic waves. The two second-harmonic signals (denoted as $\mathrm{SH} 2$ ) represent waves emitted due to the interaction of fundamental photons from each constituent fundamental beam $(\mathrm{FW})$. On the other hand, the central line (SH1) is a result of interaction of fundamental photons from 


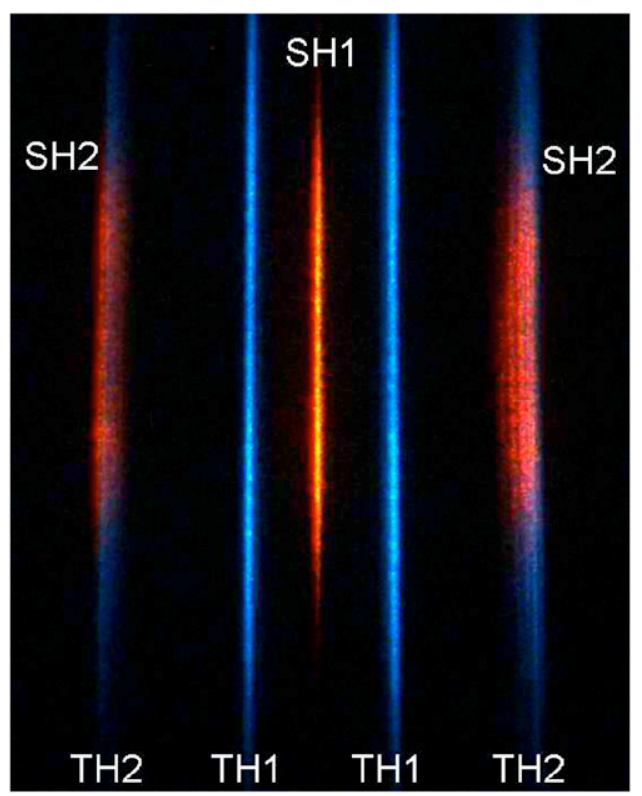

Figure 2. Experimentally recorded planar emission of the second (SH) and third (TH) harmonics.

both FWs; hence, it represents the autocorrelation signal of incoming pulses [11]. Figure 3(a) illustrates the phasematching condition for this process in the $X-Z$ plane. In this case $\vec{G}_{1}$ denotes one of the reciprocal vectors provided by the randomness. Figure 3(b) depicts the phase matching in the three-dimensional (3D) setting. The number of differently oriented reciprocal vectors $\vec{G}$ located on an arc defined by the magnitude of the wave vector $\left(\overrightarrow{K_{2} \omega}\right)$ of the SH reflects the planar emission of $\mathrm{SH}$ forming a vertical line on the screen. When such generated SH photons interact with photons from the fundamental beams via the sum-frequency mixing, the outcome is cascaded third-harmonic generation. Due to the presence of two non-collinear fundamental beams and three SH signals, the third-harmonic emission acquires the four-line pattern. Similar to SH generation, the sumfrequency mixing also utilizes the reciprocal wave vectors $G$ to fulfil its corresponding phase-matching conditions. In typical fabricated structures the simultaneous phase matching of two such processes would require careful design of the ferroelectric domain pattern and would work for only one set of frequencies. Here, the randomness ensures that there are always reciprocal vectors available which can phase match practically all quadratic processes for the broad spectrum of the fundamental waves. The emission of the four third-harmonic lines can be explained by considering again the respective phase-matching conditions of the underlying wave-mixing mechanism. In particular, the two lines denoted as TH2 are formed by the interaction of the fundamental beam with its corresponding second harmonic (SH2). On the other hand, the third-harmonic signal marked as TH1 is emitted via two different processes which are illustrated in figure 3(c). The first one involves the fundamental wave $\overrightarrow{K_{1}}$, the SH signal $\overrightarrow{K_{2 \omega}^{\prime}}$ originating from the second fundamental wave $\overrightarrow{K_{1 \omega}^{\prime}}$ and the reciprocal wave vector $\vec{G}_{4}$. The resulting third-harmonic signal is emitted in the direction indicated by the vector $\overrightarrow{K_{3 \omega}}$. However, this TH signal will also receive a contribution originating from the interaction of the auto-correlation $\mathrm{SH}$ signal (with the wave vector $\overrightarrow{K_{2} \omega}$ ) with one of the fundamental wave $\overrightarrow{K_{1 \omega}^{\prime}}$ employing the reciprocal vector $\vec{G}_{5}$. The graph in figure 3(d) illustrates the full $3 \mathrm{D}$ character of the interaction leading to the emission of the third-harmonic signal in a form of the line TH1. As can be seen in this figure, the TH emission in a particular direction is due to the contributions of the $\mathrm{SH}$ signals in all directions. Also a single SH signal will contribute to the TH emission in different spatial directions. An experimentally recorded image of the emission lines (see figure 2) shows that the spatial (vertical) extent of the third harmonic is significantly larger than that of the $\mathrm{SH}$. This is a consequence of the fact that in each of the quadratic processes such as SHG or (a)

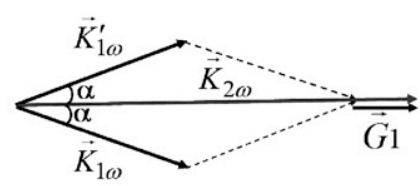

(c)

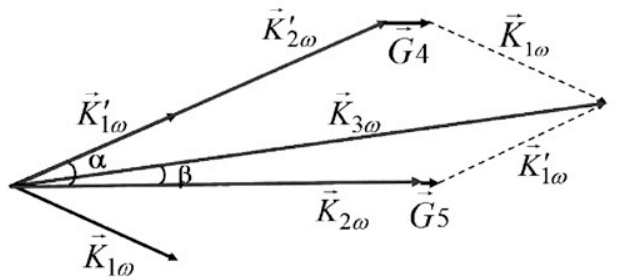

(b)

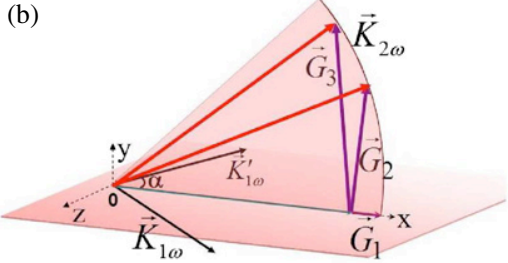

(d)

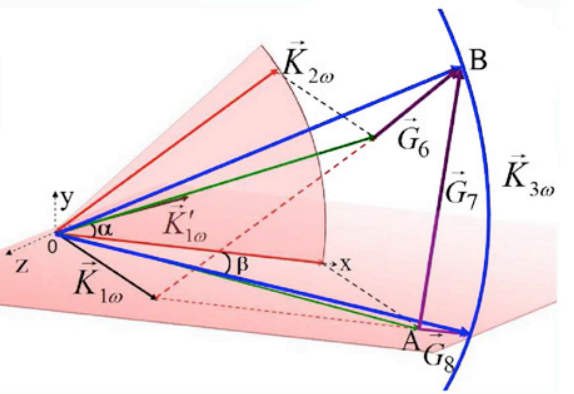

Figure 3. Diagrams illustrating the phase matching-scenarios for second- (a and b) and cascaded third-harmonic (c and d) generation in a random SBN crystal in the presence of two fundamental beams. $\vec{G}_{1} \ldots \vec{G}_{8}$ represent reciprocal vectors provided by the randomness of the nonlinearity. See the text for details. 

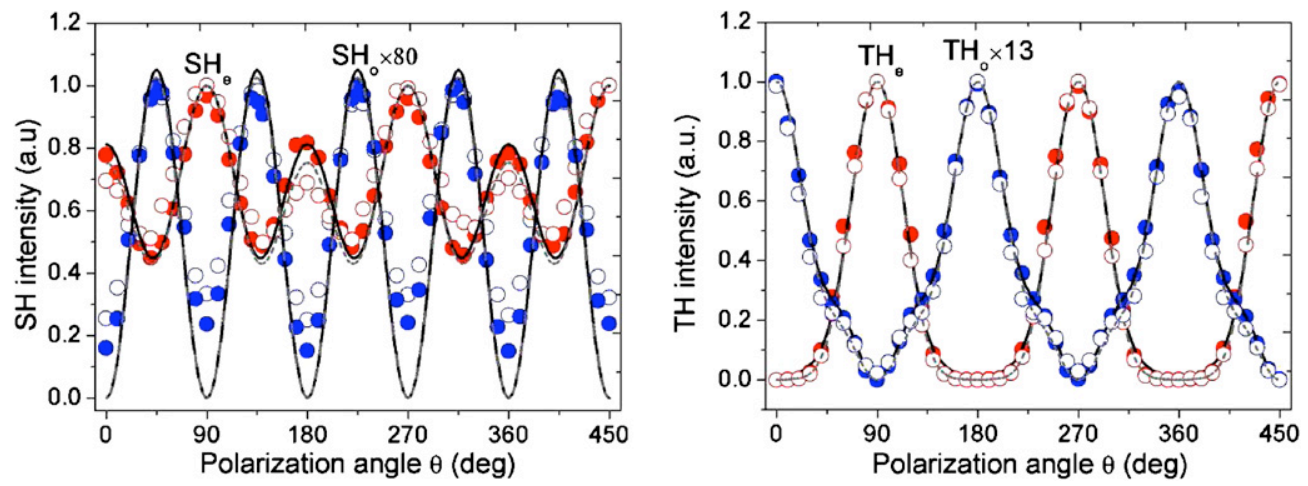

Figure 4. Intensity of the second- (left) and third-harmonic (right) signals as a function of the polarization angle of the input fundamental beams. Filled and open circles denote the experimental points for the central (SH1,TH1) and peripheral (SH2,TH2) harmonic signals, respectively. The solid line represents the theoretical fit (equations (1)-(4)). The red and blue colours refer to the extraordinary and ordinary components of the corresponding harmonics.

sum-frequency mixing the randomness of the nonlinearity results in delocalized emission of the generated frequency [1719]. As the third harmonics is formed via cascading of two quadratic processes its spatial distribution is broader than each of the constituent interactions.

The strength of the nonlinear interaction in quadratic media is determined by the effective nonlinearity which itself is a function of polarization of the interaction waves. Hence, by studying the polarization characteristics of the emitted harmonics one can extract information about details of the particular interaction process. In the experiment we varied the azimuthal angle $\theta(\theta$ is the angle with respect to the $y$-axis of the sample) of the linearly polarized fundamental wave from $0^{\circ}$ to $360^{\circ}$, while measuring the power of the extraordinary and ordinary components of the emitted harmonics. The results of these measurements are shown in figure 4, where the experimental data are represented by filled and open circles for the central (SH1,TH1) and peripheral (SH2,TH2) harmonic signals, respectively. Figures 4 (a) and (b) show the dependence of the ordinary and extraordinary components of the $\mathrm{SH}$ and $\mathrm{TH}$ on polarization of the fundamental beam. The plots show that the polarization properties of the $\mathrm{SH}$ and $\mathrm{TH}$ beams do not depend on the particular emission directions. This is because the angle between two fundamental beams is relatively small.

The solid lines in figure 4 represent the theoretically predicted dependence. It has been obtained by considering all possible processes contributing towards the second- and third-harmonic signals. Let us denote by $O_{1}$ and $E_{1}\left(O_{1}^{\prime}\right.$ and $\left.E_{1}^{\prime}\right)$ the ordinary and extraordinary components of the two fundamental waves and by $O_{j}$ and $E_{j}$ the ordinary and extraordinary components of second $(j=2)$ and third $(j=3)$ harmonics, respectively. Then, for arbitrary polarized fundamental beams, the second and third harmonics will contain ordinary and extraordinary components. In the case of e.g. SH1 second-harmonic generation, its extraordinary component is formed via the following two processes:

$$
E_{1} E_{1}^{\prime} \rightarrow E_{2} \quad \text { and } \quad O_{1} O_{1}^{\prime} \rightarrow E_{2} .
$$

On the other hand, the ordinary component of the same SH signal is obtained via the following interaction:

$$
E_{1}^{\prime} O_{1} \rightarrow O_{2} \quad \text { and } \quad E_{1} O_{1}^{\prime} \rightarrow O_{2} \text {. }
$$

The third harmonic is formed due to mixing of photons from the fundamental and $\mathrm{SH}$ beams. In particular, for the light emitted into the TH1 line, its extraordinary component is created via the following processes:

$$
\begin{array}{lll}
E_{1} E_{2 \mathrm{SH} 2} \rightarrow E_{3} & \text { and } & O_{1} O_{2 \mathrm{SH} 2} \rightarrow E_{3} \\
E_{1}^{\prime} E_{2 \mathrm{SH} 1} \rightarrow E_{3} & \text { and } & O_{1}^{\prime} O_{2 \mathrm{SH} 1} \rightarrow E_{3}
\end{array}
$$

while the ordinary component appears due to the following interaction:

$$
\begin{aligned}
& E_{1} O_{2 \mathrm{SH} 2} \rightarrow O_{3} \quad \text { and } \quad O_{1} E_{2 \mathrm{SH} 2} \rightarrow O_{3} \\
& E_{1}^{\prime} O_{2 \mathrm{SH} 1} \rightarrow O_{3} \quad \text { and } \quad O_{1}^{\prime} E_{2 \mathrm{SH} 1} \rightarrow O_{3} \text {. }
\end{aligned}
$$

In an analogous way one can identify all remaining processes contributing towards the $\mathrm{SH} 2$ and $\mathrm{TH} 2$ emissions. We have shown earlier $[10,15]$ that unlike homogeneous media where all these processes contribute coherently to the total amplitude of the generated harmonic, the randomness of nonlinearity induced by the multi-domain structure makes these constituent processes mutually incoherent. Therefore one can show that the intensities of the second and third harmonics depend on the polarization angle $\theta$ of the fundamental beam as follows:

$$
\begin{gathered}
I_{(2 \omega)}^{(e)}=I_{(\omega)}^{2}\left(\sin ^{4} \theta+R_{21} \cos ^{4} \theta\right) \\
I_{(2 \omega)}^{(o)}=I_{(\omega)}^{2}\left(R_{22} \cos ^{2} \theta \sin ^{2} \theta\right) \\
I_{(3 \omega)}^{(e)}=I_{(\omega)}^{3}\left(\sin ^{6} \theta+R_{31} \cos ^{4} \theta \sin ^{2} \theta\right) \\
I_{(3 \omega)}^{(o)}=I_{(\omega)}^{3}\left(\cos ^{6} \theta+R_{32} \cos ^{2} \theta \sin ^{4} \theta\right),
\end{gathered}
$$

where $R_{2 j}(j=1,2)$ and $R_{3 k}(k=1,2)$ denote the strengths of the constituent nonlinear processes and are determined by the corresponding effective nonlinearity [11]. It is worth noting that the effective nonlinearity for all constituent processes depends not only on the material parameters, polarization and geometry of interaction but is also affected by the disorder as has been shown by Le Grand et al [20]. We have recently extended this theory to cascaded processes [21]. It appears that disorder generally weakens the strength of the quadratic interaction and its effect depends on the phase mismatch of the process. In fitting the theoretical formulae to the 
experimental data we used the following values for the $R_{2 j}$ and $R_{3 k}$ parameters: $R_{21}=0.75, R_{22}=4.10$ and $R_{31}=0.25$, $R_{32}=1.40$ for the harmonic lines SH1 and TH1; $R_{21}=0.82$, $R_{22}=4.20$ and $R_{31}=0.04, R_{32}=1.31$ for the harmonic lines $\mathrm{SH} 2$ and $\mathrm{TH} 2$;

Finally, we investigated numerically the second- and third-harmonic generation in disordered quadratic media with two intersecting fundamental beams with Gaussian spatial and temporal profiles. Starting from Maxwell's equations and considering monochromatic stationary waves we can write the generic and well-known equation describing the electromagnetic interaction that for the transverse-electric case, for example, holds as

$$
\nabla^{2} \mathbf{E}_{j}-k_{0 j}{ }^{2} n_{j}^{2} \mathbf{E}_{j}=\mu_{0} \omega^{2} \mathbf{P}_{\mathrm{NL}, j},
$$

where $k_{0 j}=\omega_{j} / c, \omega_{j}$ is the frequency, $n_{j}$ is the index of refraction and $j=\mathrm{FB} 1, \mathrm{FB} 2, \mathrm{SH}, \mathrm{TH}$ discriminates for the two noncollinear fundamental beams, second and third harmonic respectively. The Gaussian fundamental beams, assumed to be undepleted, are tuned at $1450 \mathrm{~nm}$, are $24 \mu \mathrm{m}$ in diameter and impinge at the nonlinear medium at an internal angle $\alpha= \pm 2.35^{\circ}$ with respect to the $X$ axis. The second- and thirdharmonic nonlinear polarizations can be simply expressed as

$$
\begin{aligned}
& \mathbf{P}_{\mathrm{NL}, \mathrm{SH}}=\epsilon_{0} \chi^{(2)}(x, y)\left[\mathbf{E}_{\mathrm{FB} 1}^{2}+\mathbf{E}_{\mathrm{FB} 2}^{2}+2 \mathbf{E}_{\mathrm{FB} 1} \mathbf{E}_{\mathrm{FB} 2}\right] \\
& \mathbf{P}_{\mathrm{NL}, \mathrm{TH}}=4 \epsilon_{0} \chi^{(2)}(x, y)\left[\left(\mathbf{E}_{\mathrm{FB} 1}+\mathbf{E}_{\mathrm{FB} 2}\right) \mathbf{E}_{\mathrm{SH}}\right],
\end{aligned}
$$

where we neglected the cubic nonlinearity and did not impose any constrain on the $2 \mathrm{D}$ spatial distribution of the $\chi^{(2)}$ nonlinearity. The equations were solved with a standard 2D finite-element method considering several realizations of Gaussian distributions of the nonlinear domain pattern. As example, in figure 5 we report the case of domain distribution with $1 \mu \mathrm{m}$ mean value and $0.15 \mu \mathrm{m}$ standard deviation. Plots in figure 5 depict the far-field intensity at the exit surface of the medium of both second- and third-harmonic signals. The different emission angles are due to the noncollinear phase-matching conditions and are in good agreement with the above discussion. For the peripheral emission lines, the $\mathrm{SH}$ and TH overlap as in the experiment while for the centrally emitted (TH1) third harmonic, the simulated emission angle is $\beta=1.70^{\circ}$, corresponding very well to the measured angle of $1.75^{\circ}$. It is clear from these plots that the signals corresponding to the noncollinear interactions are always stronger than those originating from the collinear interaction. This effect, also clear from the experimental data, results from the fact that the corresponding effective second-order nonlinearity is at least four times stronger in the former case [11]. Varying the parameters of the domain distribution (i.e. mean value and standard deviation) does not affect the positions of the peaks but only changes the relative emitted intensity, since it will only change the number of $\vec{G}$ vectors available to satisfy that particular phase-matching relation.

\section{Conclusions}

In conclusion, we studied the second- and third-harmonic generation in a quadratic crystal with disordered ferroelectric domains via mixing of two fundamental beams. The

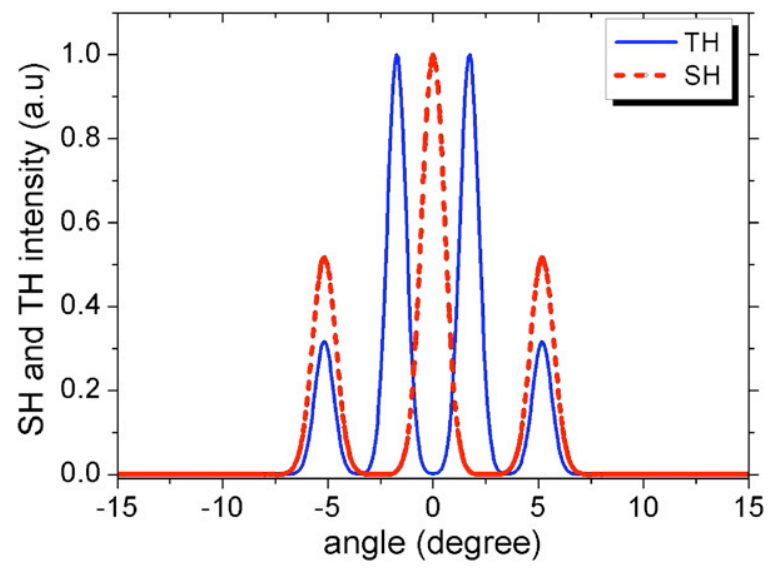

Figure 5. Numerically simulated far-field intensity of the second (red-dashed) and third (blue-solid) harmonics generated via interaction of two fundamental waves in the SBN crystal with random domain distribution.

nonlinear interaction leads to the second and third harmonics being emitted in a form of multiple lines whose positions are determined by the corresponding phase-matching condition associated with particular constituent interaction mechanism. We analysed the polarizational properties of the emitted harmonics and showed that they can be reproduced theoretically by considering effective second-order nonlinearities and taking into effect the randomness of the system which leads to incoherent character. Our experimental results agree well with the theoretical model incorporating the effect of nonlinearity disorder in the parametric wave interaction.

Since the second- and third-harmonic emission patterns depend on the randomness of the domain distribution they can be used to characterize the degree of randomness of the domain pattern in quadratic crystals. Moreover, simultaneous polarization measurements of $\mathrm{SH}$ and TH may be employed to determine the various nonlinear coefficients of the medium. In addition, as the TH1 third-harmonic signals represent actually the third-order correlation of the input pulses they can be used, together with the autocorrelation SH1 signal, in short pulse characterization and monitoring.

\section{Acknowledgments}

This work was supported by the Australian Research Council, Spanish Government (FIS2008-06024-C03-02), Catalan Government (2009 SGR 1168) and the COST Action MP0702.

\section{References}

[1] Baudrier-Raybaut M, Haidar R, Kupecek Ph, Lemasson Ph and Rosencher E 2004 Random quasi-phase-matching in bulk polycrystalline isotropic nonlinear materials Nature (London) 432374

[2] Horowitz M, Bekker A and Fischer B 1993 Broad-band 2nd-harmonic generation in $\mathrm{Sr}_{x} \mathrm{Ba}_{1-x} \mathrm{Nb}_{2} \mathrm{O}_{6}$ by spread-spectrum phase-matching with controllable domain gratings Appl. Phys. Lett. 622619 
[3] Kawai S, Ogawa T, Lee H S, DeMattei R C and Feigelson R S 1998 Second-harmonic generation from needlelike ferroelectric domains in $\mathrm{Sr}_{0.6} \mathrm{Ba}_{0.4} \mathrm{Nb}_{2} \mathrm{O}_{6}$ single crystals Appl. Phys. Lett. 73768

[4] Fejer M M, Magel G A, Jundt D H and Byer R L 1992 Quasi-phase-matched 2nd harmonic-generation-tuning and tolerances IEEE J. Quantum. Electron. 282631

[5] Molina P, Ramirez M O and Bausa L E 2008 Strontium barium niobate as a multifunctional two-dimensional nonlinear photonic glass Adv. Funct. Mater. 18709

[6] Romero J J, Jaque D, García Solé J and Kaminskii A A 2002 Simultaneous generation of coherent light in the three fundamental colors by quasicylindrical ferroelectric domains in $\mathrm{Sr}_{0.6} \mathrm{Ba}_{0.4}\left(\mathrm{NbO}_{3}\right)_{2}$ Appl. Phys. Lett. 814106

[7] Romero J J, Aragó C, Gonzalo J A, Jaque D and García Solé J 2003 Spectral and thermal properties of quasiphasematching second-harmonic generation in

$\mathrm{Nd} 3+: \mathrm{Sr}_{0.6} \mathrm{Ba}_{0.4}\left(\mathrm{NbO}_{3}\right)_{2}$ multi-self-frequency-converter nonlinear crystals J. Appl. Phys. 933111

[8] Ramirez M O, Romero J J, Molina P and Bausa L E 2005 Near infrared and visible tunability from a diode pumped $\mathrm{Nd}^{3+}$ activated strontium barium niobate laser crystal Appl. Phys. B 81827

[9] Tunyagi A R, Ulex M and Betzler K 2003 Noncollinear optical frequency doubling in strontium barium niobate Phys. Rev. Lett. 90243901

[10] Trull J, Cojocaru C, Fischer R, Saltiel S M, Staliunas K, Herrero R, Vilaseca R, Neshev D N, Krolikowski W and Kivshar Yu S 2007 Second-harmonic parametric scattering in ferroelectric crystals with disordered nonlinear domain structures Opt. Express 1515868

[11] Roppo V, Dumay D, Trull J, Cojocaru C, Saltiel S M, Staliunas K, Vilaseca R, Neshev D N, Krolikowski W and Kivshar Yu S 2008 Planar second-harmonic generation with noncollinear pumps in disordered media Opt. Express 1614192
[12] Fischer R, Neshev D N, Saltiel S M, Krolikowski W and Kivshar Yu S 2006 Broadband femtosecond frequency doubling in random media Appl. Phys. Lett. 89191105

[13] Fischer R, Neshev D N, Saltiel S M, Sukhorukov A A, Krolikowski W and Kivshar Yu S 2007 Monitoring ultrashort pulses by the transverse frequency doubling of counterpropagating pulses in random media Appl. Phys. Lett. 91031104

[14] Sheng Y, Saltiel S M and Koynov K 2009 Cascaded third-harmonic generation in single short-range-ordered nonlinear photonic crystal Opt. Lett. 34656

[15] Wang W et al 2009 Third-harmonic generation via broadband cascading in disordered quadratic nonlinear media Opt. Express 17 20117-23

[16] Sheng Y, Best A, Butt H J, Krolikowski W, Arie A and Koyonv K 2010 Three-dimensional ferroelectric domain visualization by Cherenkov-type second harmonic generation Opt. Express 1816539

[17] Roppo V, Wang W, Kalinowski K, Kong Y, Cojocaru C, Trull J, Vilaseca R, Scalora M, Krolikowski W and Kivshar Yu 2010 The role of ferroelectric domain structure in second harmonic generation in random quadratic media Opt. Express 18 4012-22

[18] Stivala S, Busacca A C, Pasquazi A, Oliveri R L, Morandotti R and Assanto G 2010 Random quasi-phase-matched second-harmonic generation in periodically poled lithium tantalate Opt. Lett. 35 363-5

[19] Pasquazi A, Busacca A, Stivala S, Morandotti R and Assanto G 2010 Nonlinear disorder mapping through three-wave mixing IEEE Photonics J. 2 18-28

[20] Grand Y Le, Rouede D, Odiu C, Aubry R and Mattauch S 2001 Second-harmonic scattering by domains in $\mathrm{RbH}_{2} \mathrm{PO}_{4}$ ferroelectric Opt. Commun. 200249

[21] Kalinowski K 2010 Second and third harmonic generation in media with random ferroelectric domains (in preparation) 